\title{
Feeding mechanisms in carp: crossflow filtration, palatal protrusions and flow reversals
}

\author{
Todd Callan
}

S. Laurie Sanderson

College of William and Mary, slsand@wm.edu

Follow this and additional works at: https://scholarworks.wm.edu/aspubs

Part of the Marine Biology Commons

\section{Recommended Citation}

Callan, Todd and Sanderson, S. Laurie, Feeding mechanisms in carp: crossflow filtration, palatal protrusions and flow reversals (2003). Journal of Experimental Biology, 206, 883-892.

doi: 10.1242/jeb.00195

This Article is brought to you for free and open access by the Arts and Sciences at W\&M ScholarWorks. It has been accepted for inclusion in Arts \& Sciences Articles by an authorized administrator of W\&M ScholarWorks. For more information, please contact scholarworks@wm.edu. 
doi:10.1242/jeb.00195

\title{
Feeding mechanisms in carp: crossflow filtration, palatal protrusions and flow reversals
}

\author{
W. Todd Callan and S. Laurie Sanderson* \\ Department of Biology, College of William and Mary, Williamsburg, VA 23187, USA \\ *Author for correspondence (e-mail: slsand@wm.edu)
}

Accepted 4 December 2002

\begin{abstract}
Summary
It has been hypothesized that, when engulfing food mixed with inorganic particles during benthic feeding, cyprinid fish use protrusions of tissue from the palatal organ to retain the food particles while the inorganic particles are expelled from the opercular slits. In crossflow filtration, the particle suspension is pumped parallel to the filter surface as filtrate exits through the filter pores, causing the suspension to become more concentrated as it travels downstream along the filter. We used high-speed video endoscopy to determine whether carp Cyprinus carpio use crossflow filtration and/or palatal protrusions

chemosensory function rather than a mechanical particlesorting function. However, palatal protrusions did retain large food particles while large inorganic particles were spit anteriorly from the mouth. We also investigated whether flow is continuous and unidirectional during suspension feeding in carp. As reported previously for ventilation in hedgehog skates and for certain industrial crossflow filtration applications, we observed that flow is pulsatile and bidirectional during feeding. These results have implications for hydrodynamic models of crossflow filtration in suspension-feeding fishes.
\end{abstract} during benthic feeding. We found that carp use crossflow filtration to concentrate small food particles in the pharyngeal cavity while expelling small dense inorganic particles through the opercular slits and via spits. Our results suggest that, during feeding on small food particles, palatal protrusions serve a localized
Movies available on-line

Key words: suspension feeding, benthic feeding, hydrodynamics, palatal organ, carp, Cyprinus carpio.

\section{Introduction}

Suspension-feeding fish filter minute prey (approximately 5-3000 $\mu \mathrm{m}$ ) from large volumes of water that enter through the mouth and exit via the opercula (Gerking, 1994; Sanderson and Wassersug, 1993). Some members of the most speciesrich freshwater fish family, the Cyprinidae, have been reported to suspension-feed by using a branchial sieve composed of gill arches with interdigitating rows of gill rakers to strain food particles from the water (Hoogenboezem et al., 1993; Van den Berg et al., 1994). In the reducible-channel model of sieving, food particles are trapped in the channels between the medial gill rakers, and the mesh size of the sieve can be reduced when the lateral gill rakers are lowered under muscular control into the channels (Hoogenboezem et al., 1991). Computational fluid dynamics and video endoscopy have indicated that the gill rakers of other suspension-feeding cyprinid species function as a crossflow filter rather than a dead-end sieve (Sanderson et al., 1991, 2001). During crossflow filtration, small food particles pass parallel to the gill arches while traveling at high velocities from the oral jaws towards the posterior oropharyngeal cavity. As the suspension moves through the pharyngeal region, filtrate exits between the gill rakers while the particles become more concentrated as they continue with the crossflow towards the esophagus (for a review, see Brainerd, 2001).

Many cyprinid species are facultative suspension feeders that can filter zooplankton and detritus as well as capture larger prey, such as chironomids, molluscs and seeds, from the substrate (García-Berthou, 2001; Lammens and Hoogenboezem, 1991). When cyprinids feed from the substrate (i.e. benthic feed), they often engulf mixtures of food and inorganic materials with the suction created by their protrusile mouth. However, cyprinids are able to separate food from inorganic particles within the oropharyngeal cavity, ejecting the inorganic particles and ingesting the food (Osse et al., 1997; Sibbing, 1988). The palatal organ, a thick muscular pad that covers the roof of the anterior pharynx in cyprinids (Matthes, 1963), is thought to be involved in the selective retention of food particles inside the oropharyngeal cavity. When taste buds in the lining of the palatal organ are stimulated, the palatal organ is hypothesized to produce local muscular projections, which pin the food particles against the gill arches while the inorganic particles are rinsed posteriorly and expelled from the opercular slits (Sibbing, 1988; Sibbing et al., 1986). 
Suspension-feeding fishes have not yet been studied to assess whether they use crossflow filtration during benthic feeding, when food particles are mixed with inorganic particles inside the oropharyngeal cavity. Our purpose is to investigate the mechanisms that are used in carp (Cyprinus carpio, Cyprinidae) to separate food and inorganic particles inside the oropharyngeal cavity and to determine whether crossflow filtration and palatal protrusions are involved. Here, we report data obtained by direct observation inside the oropharyngeal cavity using high-speed video endoscopy.

Fiberoptic endoscopy can also be used to study the patterns of flow that are generated in the oropharyngeal cavity during ventilation. Summers and Ferry-Graham (2001) observed 'flow reversals' through an endoscope during ventilation in hedgehog skates (Leucoraja erinacea). In the oropharyngeal cavity, this flow reversal consisted of a cessation of anteriorto-posterior flow followed by a brief posterior-to-anterior flow after every intake of water. In the parabranchial (opercular) cavities, the ventilatory flow stopped frequently and sometimes also reversed direction to travel from posterior to anterior. These observations of flow reversals contradict the common assumption that water flow through the oropharyngeal cavity of fishes during ventilation is generally unidirectional from anterior to posterior and continuous rather than pulsatile (Ballintijn, 1969). There have been no studies to assess whether flow is unidirectional and continuous during suspension feeding in fish. The occurrence of bidirectional or pulsatile flow could have implications for filter performance (e.g. Stairmand and Bellhouse, 1985).

In the present study, we focus on four questions. Do carp use crossflow filtration as a suspension-feeding mechanism? Do carp use the palatal organ for selective retention of particles by pinning and, if so, which particles are pinned and in what circumstances? If protrusions from the palatal organ do occur, do the protrusions always occur for the direct purpose of sorting food particles from inorganic particles? And finally, does pulsatile or bidirectional flow occur in carp during feeding?

\section{Materials and methods}

Cyprinus carpio L. (Israeli carp) were obtained from a local aquaculture company. The carp were maintained on a Tetramin flake diet while held individually in 110-liter aquaria at room temperature $\left(21^{\circ} \mathrm{C}\right)$ with a substrate of either medium-grain quartz sand $(0.5-1.5 \mathrm{~mm}$ diameter $)$ or gravel $(0.3-1.0 \mathrm{~cm}$ diameter). Endoscopy experiments were performed on five specimens (26.5-29.5 cm standard length) using methods similar to those described in Sanderson et al. (1996, 2001). Each carp was anesthetized with MS-222, and a polyethylene cannula (45 cm long, $2.15 \mathrm{~mm}$ i.d., $3.25 \mathrm{~mm}$ o.d., Intramedic PE 280) was inserted into the oropharyngeal cavity through a hole drilled in the left or right preopercular bone. A flange (approximately $1 \mathrm{~mm}$ wide) around the circumference of one end of the cannula lay flush against the tissue of the oropharyngeal cavity, preventing the cannula from being pulled through the hole. The cannula fitted tightly into the drilled hole, eliminating any flow of water through the hole around the cannula. The external part of the cannula was then threaded through a second flanged polyethylene cannula (2.5 cm long, $3.76 \mathrm{~mm}$ i.d., $4.82 \mathrm{~mm}$ o.d., Intramedic PE 360) to prevent the cannula from slipping back into the oropharyngeal cavity. A small neoprene rubber pad $(0.8 \mathrm{~cm} \times 0.8 \mathrm{~cm})$ was placed between the second flange and the skin to reduce irritation. The fish was then returned to the aquarium. At the conclusion of the experiment, the cannula was removed while the fish was under anesthesia. Subsequently, the insertion site healed completely.

After approximately $4 \mathrm{~h}$ of cannula insertion, a flexible fiberoptic endoscope (Olympus ultrathin fiberscope type 14, $1.4 \mathrm{~mm}$ o.d., $1.2 \mathrm{~m}$ working length, $75^{\circ}$ field of view, $0.2-5.0 \mathrm{~cm}$ depth of field) was threaded through the cannula to the opening in the oropharyngeal cavity. The endoscope was attached to a CCD video camera (Canon Ci-20R, 30 frames s$^{-1}$ ) or a Kodak Ektapro Hi-Spec Motion Analyzer 1012/2 with an Intensified Imager VSG (50-500 frames $\mathrm{s}^{-1}$ ). A high-intensity light source (Olympus Helioid ALS-6250, 250 W) provided light to the endoscope. The video equipment was then attached to a Hi-8 video player/recorder (Sony EVO-9700).

Data were collected during feeding on bass pellets $(0.6 \mathrm{~cm}$ diameter) or a slurry of finely crushed Tetramin flakes mixed with water (particles $0.1-1.0 \mathrm{~mm}$ diameter). The pellets were mixed by hand in the gravel on the bottom of the aquarium. The Tetramin slurry was placed in the water above the fish through a short piece of tubing attached to a $30 \mathrm{ml}$ syringe. Slurry particles were engulfed by the carp as they descended through the water or lay on the substrate. The pellets and the Tetramin slurry could be discerned clearly through the endoscope. Due to their narrow size range, brine shrimp cysts (Artemia sp., $255 \pm 15 \mu \mathrm{m}$, mean \pm S.D., range $210-300 \mu \mathrm{m}$; Sanderson et al., 1998) were introduced with the slurry as tracer particles of known size. The approximate magnitudes of the flow reversals were quantified using these brine shrimp cysts. The number of diameters traveled in an anterior direction by a brine shrimp cyst during a flow reversal was recorded and converted to absolute distance using the mean diameter of $255 \mu \mathrm{m}$.

Additional endoscopy was performed on a dead specimen for confirmation of oropharyngeal structures identified in the endoscopic view. A Sony EVO-9700 Hi-8 player/recorder with a jog/shuttle was then used for frame-by-frame analysis of the videotapes. The video images used for publication were digitized using an Apple Macintosh G3. The digitized images were processed by convolving them with a mean kernel $(3 \times 3$ pixels or $5 \times 5$ pixels) using $\mathrm{NIH}$ Image 1.61 , which reduced the fine honeycomb pattern produced by the individual optical fibers in the fiberoptic bundle.

\section{Results}

Endoscopic view of the oropharyngeal cavity

From the preopercular insertion site, the endoscope entered the anterior pharynx approximately $5.0 \mathrm{~cm}$ posterior to the oral jaws and immediately lateral to the palatal organ. The 
Fig. 1. Schematic of the carp oropharyngeal cavity, indicating endoscope insertion site. The roof of the oropharynx is illustrated on the left, with the palatal organ (po) shown in coarse stippling and the region of po observed through the endoscope in fine stippling. The floor of the oropharynx is illustrated on the right, with ceratobranchials I-IV (cb I-IV) shown as black bars. The location of the gill rakers is shown by the gray shading. The hatched region of the gill arches was observable through the endoscope. Modified from Sibbing et al. (1986).

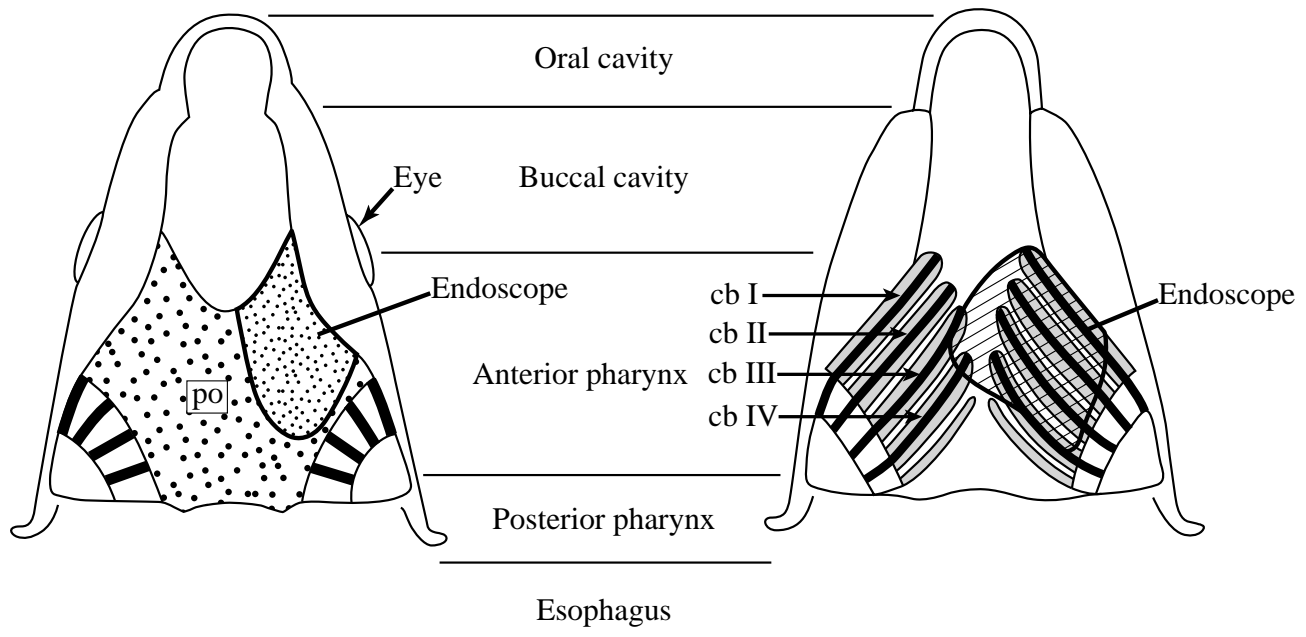

ceratobranchials of arches I-III could be seen clearly, and arch IV could be seen intermittently (Figs 1,2). Occasionally, the arches on the opposite side of the oropharyngeal cavity could be seen in the background during feeding.

\section{Intake}

Cyprinus carpio fed on all food particles by using 'slow suction', which occurs in cyprinids swimming at low velocity and capturing smaller, less mobile prey (Sibbing, 1991). This slow suction creates an overall anterior-to-posterior flow in the oropharyngeal cavity. To engulf multiple food particles suspended in the water or whirled up off the substrate, fish used a repetitive slow suction termed 'gulping' by Sibbing et al. (1986). To engulf pellets that were on or in the gravel substrate, fish used a type of slow suction termed 'particulate intake' in which a higher-velocity suction flow is usually directed towards an individual food particle (Sibbing et al., 1986).

\section{Suspended food particles}

Endoscopic videotapes were taken at $125-500$ frames s $^{-1}$ while carp were using gulping to feed on slurry particles that were suspended in the water. In the anterior pharynx, particles moved independently of each other, and no boluses of particles were observed. 100 suspended slurry particles consumed by each of five carp specimens were chosen randomly and then followed frame-by-frame as each particle passed through the endoscopic field of view ( $N=100$ slurry particles per individual fish). The vast majority of these particles $(97.6 \pm 4.3$, mean \pm S.D., $N=5$ individuals) traveled posteriorly without coming into contact with any pharyngeal surface. The remainder of the observed particles bounced once off the ceratobranchials and continued posteriorly towards the esophagus. No mucus strings or aggregates were observed in the endoscopic videotapes.

\section{Small food particles mixed with sand}

Endoscopic videotapes were taken at $125-500$ frames s $^{-1}$ while carp were using gulping to benthic feed on slurry particles off a sand substrate. The vast majority of the slurry particles traveled independently of each other in a posterior direction through the field of view without contacting any pharyngeal surface, as described above for suspended slurry particles. While the slurry remained suspended as it passed through the anterior pharynx, the sand grains that had been engulfed with the slurry had sunk towards the gill arches and generally traveled ventral to the slurry. 100 sand grains engulfed with slurry by each of three carp specimens were chosen randomly and then followed frame-by-frame as each sand grain passed through the field of view ( $N=100$ sand grains per individual fish). Most of these sand grains (78.7 \pm 1.2 , mean \pm S.D., $N=3$ individuals) rolled posteriorly along ceratobranchials I-IV, remaining less than one sand-grain diameter above these surfaces. Some sand grains $(12.0 \pm 1.0)$ were observed to bounce off the ceratobranchials and then continue their posterior travel with a mean height of $1.1 \pm 0.4$ sand-grain diameters (mean \pm S.D., $N=36$ sand grains) above these surfaces. Other sand grains $(9.3 \pm 0.6)$ were not seen to

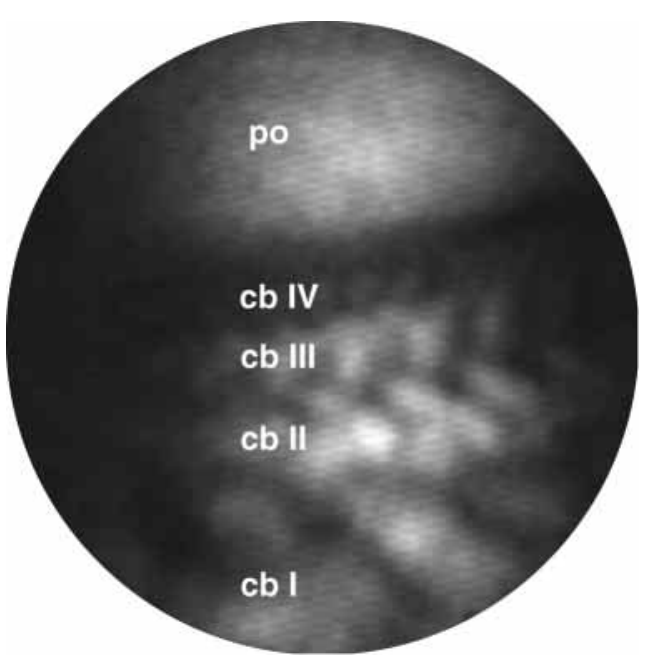

Fig. 2. Endoscopic video image showing rows of gill rakers on ceratobranchials I-IV (cb I-IV). The anterior of the fish is to the right. The palatal organ (po) on the roof of the anterior pharynx is located at the top of the image. The portion of $\mathrm{cb}$ III that is in the field of view is approximately $1.5 \mathrm{~cm}$ in length. 
bounce but simply passed through the field of view with a mean height of $1.3 \pm 0.5$ sand-grain diameters (mean \pm S.D., $N=28$ sand grains) above the ceratobranchials. Sand grains were observed to exit through the opercular slits as well as to be spat out periodically from the mouth.
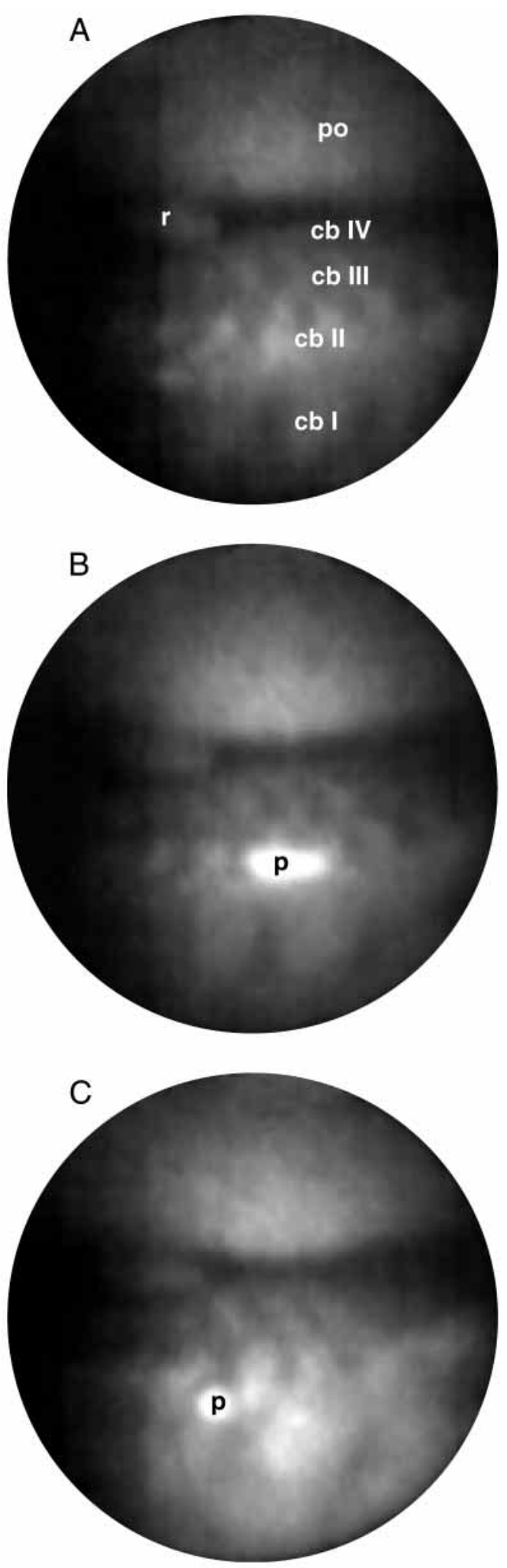

\section{Small food particles mixed with gravel}

When slurry particles sank to the bottom of an aquarium with a gravel substrate, rocks were engulfed during benthic feeding. In 5 continuous minutes of feeding on slurry off the gravel substrate by each of three carp specimens, $97.0 \pm 3.8 \%$ (mean \pm S.D., $N=3$ individuals) of the 124 rocks that were observed in the endoscopic videotapes (30-500 frames $\mathrm{s}^{-1}$ ) were pinned between the palatal organ and the gill arches by an overall height reduction of the pharyngeal slit between these structures (Fig. 3). This narrowing of the pharyngeal slit was caused primarily by ventral movement of the palatal organ. This prevented further movement of the rocks but allowed the slurry particles to continue posteriorly as described above for suspended slurry. The few rocks that entered the endoscopic field of view during feeding but were not pinned between the palatal organ and the gill arches simply exited from view in a posterior direction. Subsequently, rocks were seen to be spat out anteriorly from the oropharyngeal cavity but were never expelled from the opercular slits.

\section{Palatal protrusions during feeding on small food particles}

Protrusions of tissue from the palatal organ were observed in five specimens during feeding on suspended slurry particles or when gulping slurry off a sand or gravel substrate. These protrusions were distinct from overall height reductions of the pharyngeal slit. Using the diameter of a typical sand grain $(1.0 \mathrm{~mm})$ to estimate protrusion size from endoscopic videos in which protrusions occurred during feeding on slurry mixed with sand, the base of the protrusions at the palatal organ was calculated as $4.4 \pm 0.8 \mathrm{~mm}$ in diameter (mean \pm S.D., $N=10$ protrusions).

In $4.5 \mathrm{~min}$ of feeding on slurry off a sand substrate (125-500 frames s ${ }^{-1}$ ) by the specimen in which we observed the highest frequency of occurrence of these protrusions, 27 of 28 protrusions that occurred were in contact with the ceratobranchials of arches II, III or IV (Fig. 4) for $54 \pm 19 \mathrm{~ms}$ (mean \pm S.D.). The remaining protrusion was in contact with a ceratobranchial for the greatest length of time, $168 \mathrm{~ms}$. The brevity of contact between these protrusions and the gill arches is illustrated by comparison of the mean duration of contact with the mean duration of water intake. The mean ( \pm S.D.) duration of water intake from the start of each suction during which a protrusion was observed to the start of the next suction was $410 \pm 87 \mathrm{~ms}$ ( $N=27$ intakes; two protrusions occurred at the end of a single intake).

Fig. 3. Endoscopic images illustrating a typical sequence observed during feeding on slurry particles off a gravel substrate (125 frames s${ }^{-1}$; duration of sequence $104 \mathrm{~ms}$; frames 1,6 and 13 shown). The anterior of the fish is to the left. Rows of gill rakers on ceratobranchials I-IV (cb I-IV) are visible, as well as the palatal organ (po). (A) A rock (r) is pressed down and pinned by the palatal organ across cb IV. (B) A slurry particle (p) and (C) a brine shrimp cyst (p) travel posteriorly past the rock and do not come into contact with any pharyngeal surface while in the field of view. Movie available online (movie: fig3.mov). 

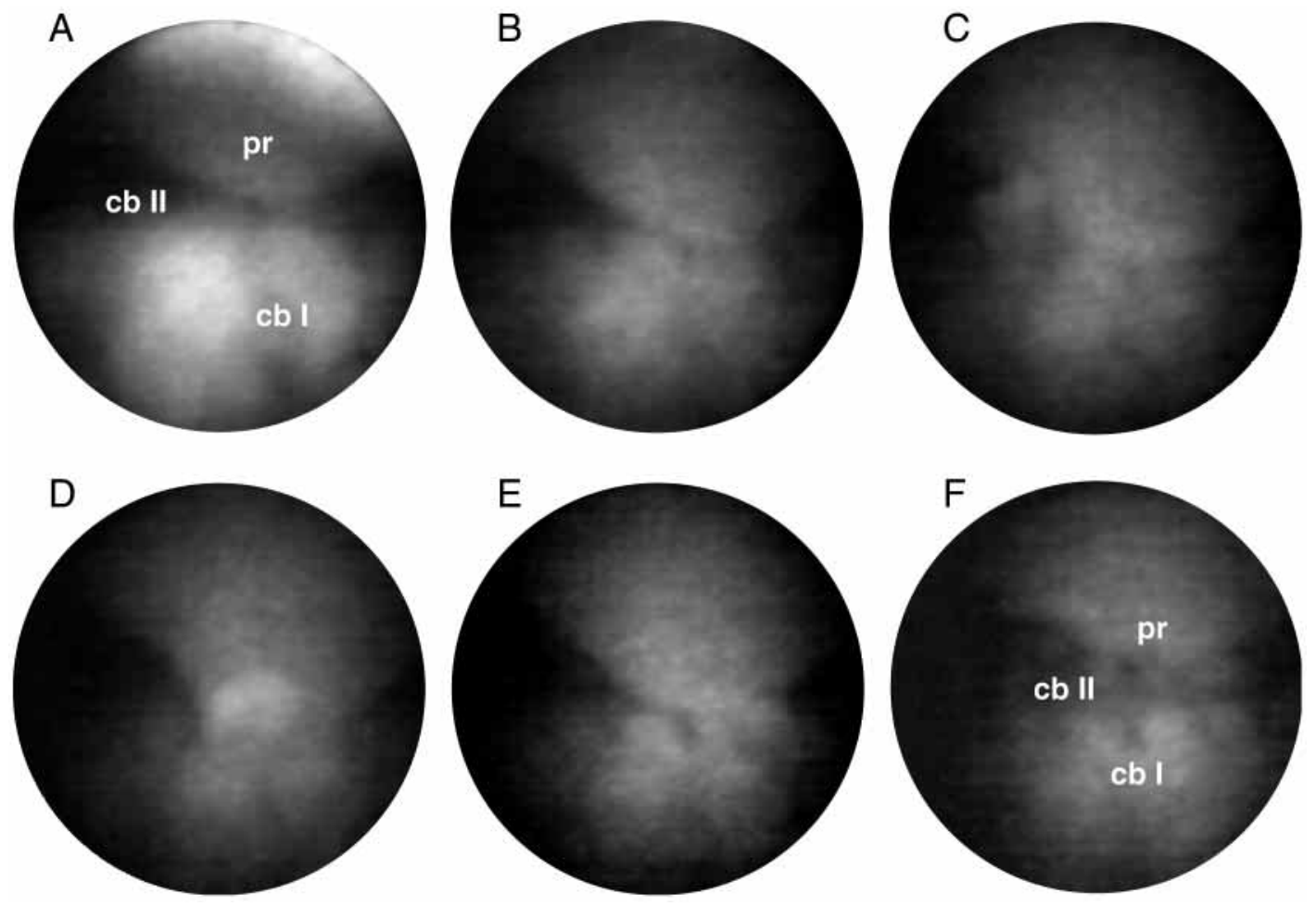

Fig. 4. Endoscopic images illustrating a protrusion (pr) of the palatal organ during feeding on slurry off a sand substrate $\left(500\right.$ frames $^{-1}$; duration of sequence $64 \mathrm{~ms}$; frames 1, 5, 8, 9, 10 and 12 shown). The anterior of the fish is to the right. Ceratobranchials I and II (cb I; cb II) are visible. (A) A protrusion of tissue from the palatal organ is projecting towards cb II. (B) The protrusion has come into contact with cb II. (C) A sand grain has entered the field of view on the left side of the protrusion during a flow reversal. (D) The sand grain passes lateral to the protrusion. (E) The protrusion begins to move dorsally and lifts from cb II while the water is still moving anteriorly during the flow reversal. (F) The palatal organ is returning to its original shape. Movie available online (movie: fig4.mov).

These protrusions occurred only after the anterior-toposterior water flow had ceased at the end of each intake or during the early stages of the posterior-to-anterior flow, which characterized a flow reversal (see below). Some protrusions were observed to pin a slurry particle or a sand grain but, due to the relatively large sizes of the protrusions compared with the sizes of the slurry and sand, we could not determine whether particles were always pinned by these protrusions. However, in the $4.5 \mathrm{~min}$ of feeding on slurry mixed with sand during which 28 protrusions were observed, more than 870 slurry particles passed through the field of view without being contacted by protrusions. As described above during feeding on slurry mixed with gravel, rocks were pinned by an overall height reduction of the pharyngeal slit rather than by these brief protrusions.

\section{Large food particles mixed with gravel}

In endoscopic videotapes of feeding on pellets mixed with gravel for five continuous minutes by each of two specimens (30-500 frames s $\left.{ }^{-1}\right)$, an overall height reduction of the pharyngeal slit, which was similar to that found during feeding on slurry mixed with gravel, was seen. The height of the pharyngeal slit varied within individuals from approximately $1 \mathrm{~mm}$ to $15 \mathrm{~mm}$. Early in feeding sequences on pellets mixed with gravel, ventral movement of the palatal organ stopped both the rocks and the pellets ( $N=22$ pellets) from continuing posteriorly. However, after the rocks and pellets were pressed simultaneously between the palatal organ and the ceratobranchials of arches II-IV, the palatal organ always moved dorsally to allow the rocks to be spit anteriorly while keeping the pellets pinned in place with protrusions of tissue from the palatal organ (Fig. 5). Using the diameter of the pellets $(0.6 \mathrm{~cm})$ to estimate protrusion size, the base of these protrusions at the palatal organ was calculated as $1.1 \pm 0.2 \mathrm{~cm}$ in diameter (mean \pm S.D., $N=10$ protrusions). The mean ( \pm S.D.) duration of contact between the palatal organ and the pellet was $809 \pm 688 \mathrm{~ms}$ (range $367-3100 \mathrm{~ms}, N=20$ protrusions).

After the gravel had been expelled, the pellets were manipulated in the anterior pharynx. This manipulation involved a height reduction of the pharyngeal slit to press a pellet against the ceratobranchials during posterior water flow or during anterior water flow caused by protrusion of the upper jaws with the mouth closed. The subsequent dorsal movement of the palatal organ released the pellet at a time that generally corresponded to a pause in the water flow during a change in flow direction from posterior to anterior or vice versa. However, the timing of pellet release was often slightly early, causing the pellet to be moved slightly posteriorly or anteriorly in the pharynx by the decelerating water flow. Degradation of the softening pellet could often be observed. This manipulation 
eventually ended when the posterior water flow moved the pellet out of the field of view towards the posterior pharynx for ingestion.

\section{Flow reversals}

A flow reversal occurred as slurry particles ceased their posterior movement through the endoscopic field of view, moved anteriorly and then stopped. The subsequent intake of water returned particles to a posterior trajectory. Flow reversals were distinct from closed protrusions. Protrusions of the premaxillae while the oral jaws remained closed ('closed protrusions'; Sibbing, 1988; Sibbing et al., 1986) occurred intermittently. Closed protrusions created extensive posteriorto-anterior water flow, usually sending particles out of the field of view into the orobuccal cavity. Since particles generally remained in the field of view during flow reversals, closed protrusions and flow reversals could be distinguished by the extent of particle movement.

Flow reversals were observed during feeding on suspended slurry particles or when gulping slurry off a sand or gravel
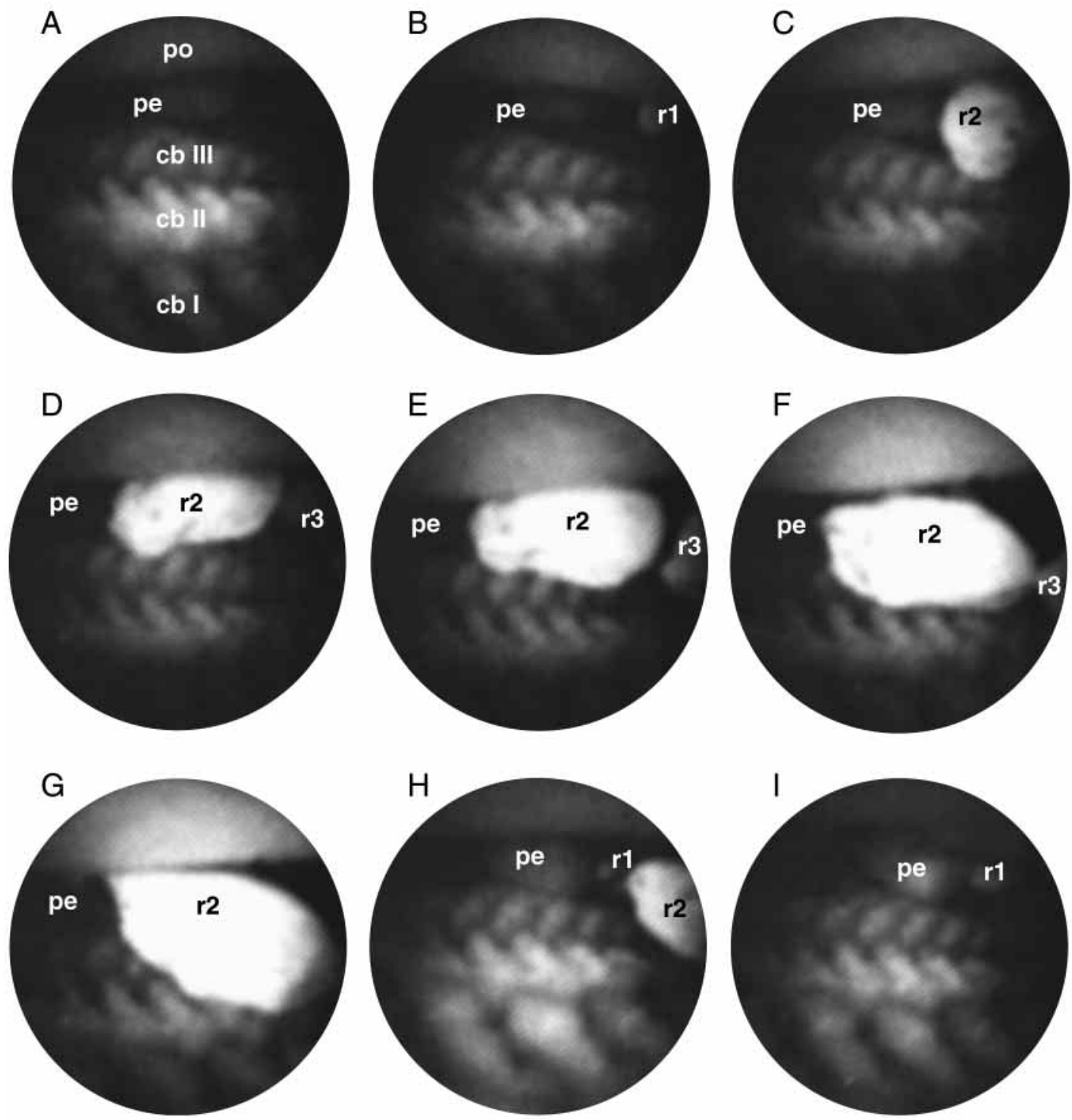

Fig. 5. Endoscopic images illustrating a typical sequence observed during feeding on pellets off a gravel substrate ( $30 \mathrm{frames}^{-1}$; duration of sequence $267 \mathrm{~ms}$ ). The anterior of the fish is to the right. The palatal organ (po) is visible above rows of gill rakers on ceratobranchials I-III (cb I-III). (A) A pellet (pe) is observed entering the field of view and being pinned across cb III by an overall height reduction of the pharyngeal slit. (B) A rock (r1) enters the field of view, followed by (C) a second rock (r2) and (D) a third rock (r3). (E) r2 is pinned across cb II by an overall height reduction of the pharyngeal slit, preventing further posterior movement. (F) r2 is released by the palatal organ. (G) r3 has moved anteriorly out of the field of view during a spit, and $\mathrm{r} 2$ moves further anteriorly. $(\mathrm{H}) \mathrm{r} 2$ almost completely leaves the field of view in an anterior direction. $\mathrm{r} 1$ is visible again behind $\mathrm{r} 2$. (I) $\mathrm{r} 2$ is spat anteriorly out of the field of view while the pellet and r1 remain pinned across cb III. $\mathrm{r} 1$ exited anteriorly from the field of view in the subsequent frame, while the pellet was retained by a palatal protrusion. Movie available online (movie: fig5.mov). 
substrate. We could not determine with certainty whether flow reversals occurred during feeding on pellets off a gravel substrate because there was an insufficient number of particles suspended in the anterior pharynx to examine frame-by-frame.

In 50 consecutive slow suctions performed by each of four specimens (125-500 frames s ${ }^{-1}$ ) during feeding on suspended slurry, a flow reversal occurred immediately after $95.5 \% \pm 3.0 \%$ (mean \pm S.D., $N=4$ individuals) of the slow suctions. Even when a flow reversal did not occur at the end of a slow suction, a cessation in the posterior flow of water through the anterior pharynx was always observed.

The duration of 10 flow reversals in each of four specimens was calculated as the time between the frame in which posterior-to-anterior flow began and the last frame in which posterior-to-anterior flow occurred. This flow reversal duration was $89 \pm 13 \mathrm{~ms}$ (mean \pm S.D., $N=4$ individuals). For comparison, the duration of the intakes during which these flow reversals occurred was calculated as the time between the frame in which anterior-to-posterior flow began to the last frame in which posterior-to-anterior flow occurred. This intake duration was $429 \pm 132 \mathrm{~ms}$ (mean \pm S.D., $N=4$ individuals).

The distance traveled by brine shrimp cysts during 10 flow reversals in each of four specimens was $4.4 \pm 0.6$ brine shrimp cyst diameters (mean \pm S.D., $N=4$ individuals). Using a mean diameter of $255 \mu \mathrm{m}$ (Sanderson et al., 1998), the approximate distance traveled by brine shrimp cysts during flow reversals was calculated as $1.1 \pm 0.2 \mathrm{~mm}$.

\section{Discussion \\ Suspended food particles}

Slurry particles remained suspended inside the oropharyngeal cavity and passed posteriorly through the anterior pharynx while the fish were feeding. Particles were not retained in the channels between gill rakers, and the gill arches and gill rakers were not utilized as a dead-end sieve by $C$. carpio. Since there were no mucus strings or aggregates, mucus entrapment of particles by aerosol filtration mechanisms (Sanderson et al., 1996) did not occur in the anterior pharynx. These data are consistent with the use of crossflow filtration as described for gizzard shad (Dorosoma cepedianum; Clupeidae), ngege tilapia (Oreochromis esculentus; Cichlidae), goldfish (Carassius auratus; Cyprinidae) and blackfish (Orthodon microlepidotus; Cyprinidae) (Goodrich et al., 2000; Sanderson et al., 2001). The lack of mucus entrapment in the anterior pharynx supports the suggestion of Beveridge et al. (1991) that bacteria were retained by drinking rather than by suspension feeding during their laboratory experiments with carp.

Crossflow filtration does not preclude the use of mucus in the posterior pharynx to aggregate concentrated food particles for transport into the esophagus, as proposed by Sibbing and Uribe (1985). We were unable to view the posterior pharynx (i.e. the chewing cavity where the pharyngeal jaws are located) through the endoscope (Fig. 1).

Microanatomical studies by Van den Berg et al. (1994) identified $\mathrm{m}$. abductores branchiospinales (MABs) on the lateral sides of gill rakers on arches I, II, III and IV in C. carpio. Their preliminary stimulation experiments in common bream (Abramis brama; Cyprinidae) indicated that contraction of the MABs abducts the lateral gill rakers, causing the lateral rakers to move into the channels between medial rakers on the adjacent gill arch. Van den Berg et al. (1994) hypothesized that C. carpio uses this mechanism to sieve small food particles (approximately 250-500 $\mu \mathrm{m}$ ) in accordance with the reduciblechannel model of filter feeding (Hoogenboezem et al., 1991, 1993). We did not observe abduction or adduction of gill rakers during feeding in C. carpio, including during suspension feeding on slurry particles ranging from approximately $100 \mu \mathrm{m}$ to $1000 \mu \mathrm{m}$ in diameter. In the endoscopic videotapes, the gill rakers moved with the gill arches as the arches abducted and adducted, but we did not observe the gill rakers moving independently of the gill arches.

\section{Food particles smaller than inorganic particles}

During benthic feeding, carp used slow suction to engulf both food and inorganic particles. When food particles were smaller and less dense than the inorganic particles with which the food was mixed, the food particles were retained by crossflow filtration as described above for suspended slurry particles. This use of crossflow filtration allowed large numbers of food particles to be separated rapidly from large numbers of inorganic particles, while simultaneously transporting the food particles to the posterior pharynx for ingestion.

In the case of numerous small inorganic particles such as sand, the sand grains sank ventral to the slurry inside the oropharyngeal cavity and rolled in a posterior direction along the ceratobranchials. The suspended slurry continued towards the posterior pharynx while sand exited posteriorly from the opercular slits and was spat periodically in an anterior direction out of the mouth. The sand that exited from the opercular slits may have passed primarily through the first branchial slit between the wall of the oropharynx and the first gill arch, as suggested by Sibbing et al. (1986). The sand that was eventually spat from the mouth may have been those grains that were initially observed to roll along the ceratobranchials in a posterior direction.

In the case of large inorganic particles such as gravel, an overall height reduction of the pharyngeal slit prevented further posterior movement of the rocks (Fig. 3). The suspended slurry traveled past the rocks towards the posterior pharynx. All rocks were then spat anteriorly from the mouth. An overall height reduction of the pharyngeal slit, caused by dorsal gill arch muscles that move the palatal organ as a whole, was predicted by Sibbing and Uribe (1985) on the basis of morphological studies.

Sibbing and Uribe (1985) described a thin layer of muscle on the pharyngeal surfaces of carp gill arches and pointed out that these muscles could participate in selective particle retention. However, we did not observe any movement, such as bulges or ridges, in this layer of muscle on the gill arches. 


\section{Palatal protrusions during feeding on small food particles}

On the basis of extensive anatomical, electromyographic and light and X-ray cinematographic studies, the separation of food and inorganic particles has been hypothesized to occur mainly in the anterior pharynx of carp, where protrusions of tissue from the palatal organ have been proposed to pin the food particles and allow the inorganic particles to be expelled from the branchial and opercular slits (Sibbing, 1988; Sibbing et al., 1986). Palatal protrusions have been observed in anesthetized or freshly killed carp as a result of mechanical, chemical or electrical stimulation of the palatal organ (Osse et al., 1997; Sibbing et al., 1986).

Our endoscopic videotapes of carp benthic feeding on large organic particles support this previously published hypothesis of food particle retention by palatal protrusions (see below). However, our data indicate that protrusions of the palatal organ do not serve to retain small food particles such as slurry. Several lines of evidence support our assertion that the small, brief palatal protrusions that we observed during feeding on small food particles (Fig. 4) do not function to separate food and inorganic particles. First, these protrusions were recorded during feeding on slurry that was mixed with sand or with gravel as well as during feeding on slurry that was suspended in the water. Thus, these protrusions occurred even when inorganic particles were not present in the oropharyngeal cavity such that food did not need to be separated from inorganic particles. Second, the most frequent occurrence of these protrusions in the endoscopic videotapes involved only 28 protrusions during $4.5 \mathrm{~min}$ of feeding. During this time, more than 870 slurry particles passed through the endoscopic field of view without being contacted by protrusions. Thus, these protrusions were rare relative to the number of food particles present in the oropharyngeal cavity. Third, these protrusions were extremely brief (approximately $55 \mathrm{~ms}$ in duration) relative to the retentive protrusions that pinned large food particles during benthic feeding off a gravel substrate (range of 367-3100 ms duration). Protrusions during feeding on small food particles occurred only at the end of an intake when all flow had stopped in the anterior pharynx or when a flow reversal was beginning. We are unable to explain how such brief protrusions during periods of no flow to slow reversed flow in the anterior pharynx could serve to sort food from inorganic particles.

We hypothesize that the palatal protrusions that we observed during feeding on small food particles serve a tasting function rather than a direct sorting function. The palatal organ in the anterior pharynx has the highest density of taste buds found in the oropharyngeal cavity (Sibbing and Uribe, 1985). Brief localized protrusions from the palatal organ at the end of an intake could provide chemosensory information on the palatability of the particles in the anterior pharynx.

Minute papillar projections (approximately $150 \mu \mathrm{m} \times 550 \mu \mathrm{m})$ of the palatal organ were described in Sibbing and Uribe's histological and electron microscopical studies of carp oropharyngeal structures (Sibbing and Uribe, 1985). We could not visualize these papillae in the endoscopic videotapes. Such papillae could play a chemosensory or mechanical role after contact of a palatal protrusion with the ceratobranchials. However, our endoscopic measurements of the pharyngeal slit $(1-15 \mathrm{~mm})$ during feeding indicate that the papillae are too short (up to $150 \mu \mathrm{m}$ high; Sibbing and Uribe, 1985) to contact the ceratobranchials in the absence of a palatal protrusion.

\section{Large food particles and inorganic particles}

Our data indicate that when food particles are too large to be crossflow filtered and are mixed with inorganic particles in the anterior pharynx, individual food particles (e.g. pellets) are retained by palatal protrusions while the inorganic particles (e.g. rocks) are spat anteriorly out of the mouth (Fig. 5). The occurrence of palatal protrusions to selectively retain food particles in carp has been hypothesized previously (Sibbing, 1988; Sibbing et al., 1986). Our endoscopic observations differ from these hypotheses in that palatal protrusions were observed to selectively retain food particles only when the food particles were too large to be separated from the inorganic particles by crossflow filtration.

Horseradish peroxidase studies of the vagal gustatory system in goldfish and some carp species have demonstrated a sensorimotor mapping of the palatal organ in the vagal lobe of the medulla (Finger, 1988, 1997). Sensory neurons are connected via interneurons to underlying motor neurons that innervate the stimulated region of the palatal organ. Consequently, when a palatal protrusion contacts a palatable particle, an oropharyngeal reflex could cause the protrusion to continue to pin the particle against the gill arches, whereas contact with an inorganic particle could result in release of the particle so that it could be spat anteriorly (Finger, 1997).

Peristaltic waves of muscular contraction in the palatal organ have been proposed to propel food to the chewing cavity (Sibbing, 1988; Sibbing et al., 1986). In the regions of the anterior pharynx that were observed through the endoscope (Fig. 1), waves of muscular contraction were not observed. In the endoscopic videotapes, pellets were seen to be transported via water flow in the anterior pharynx. The manipulation of pellets that was recorded in the anterior pharynx after the rocks had been spat anteriorly out of the mouth may have served two functions: (1) to confirm that palatable particles had been sorted from inorganic particles and (2) to hold pellets while other pellets were being processed by the pharyngeal jaws in the posterior pharynx.

\section{Flow reversals}

Protrusions of the premaxillae while the oral jaws remained closed (closed protrusions) resuspended particles in the orobuccal cavity (Sibbing, 1988; Sibbing et al., 1986) and may have aided in the separation of food and inorganic particles (e.g. by resuspending slurry that had been trapped between sand grains). The winnowing behavior described by Drucker and Jensen (1991) in embiotocids was similar to a closed protrusion, except that the oral jaws remained slightly open during winnowing. 
Although closed protrusions and flow reversals occurred at the same stage during feeding (i.e. at the end of an intake), closed protrusions were intermittent whereas flow reversals were observed at the end of approximately $95 \%$ of the intakes during feeding on suspended slurry. Even when the flow did not actually reverse at the end of an intake, a cessation of anterior-to-posterior flow was always recorded in the anterior pharynx. Similar flow reversals were reported in hedgehog skates by Summers and Ferry-Graham (2001) during a mode of ventilation termed 'mouth + spiracle'. We have also observed brief periods of slight posterior-to-anterior flow in the pharynx of ngege tilapia during ventilation and during suspension feeding (S.L.S. and W.T.C., personal observation).

The cause of such flow reversals and whether they serve a role in ventilation and suspension feeding or are simply a consequence of the kinematics and biomechanics of the system are unknown. Observations by Summers and Ferry-Graham (2001) suggested that water actually travels from the parabranchial chambers into the oropharyngeal cavity during a flow reversal. Experiments are planned to determine whether flow reversals during suspension feeding result from (1) the passage of water through the branchial slits in a posterior-toanterior direction, (2) secondary flow caused by the obstruction that is formed when the gill arches adduct and/or (3) slight shape changes within the oropharyngeal cavity.

As discussed by Summers and Ferry-Graham (2001), the occurrence of flow reversals has important implications for gas transfer models that generate predictions of oxygen extraction efficiencies. Similarly, flow reversals have potential impacts on hydrodynamic modeling of filtration in suspension-feeding fishes. A major goal of industrial crossflow filtration engineers is the reduction of solute concentration near the membrane filter surface as well as subsequent solute deposition on the filter. These concentration polarization and fouling phenomena constitute the primary limitations to performance in pressuredriven membrane filtration systems (e.g. Brewster et al., 1993). Thus, designs that generate fluid instabilities that restrict solute build-up at the filter surface are sought. Pulsating flow through tubes with furrowed walls results in mainstream flow reversals that cause minute vortices to be ejected from the furrows into the mainstream flow, dramatically increasing filtration rates (e.g. Sobey, 1980; Stairmand and Bellhouse, 1985). While we did not observe large-scale vortices in the anterior pharynx, small-scale vortices at the level of the channels between gill rakers or the branchial slits between gill arches would be difficult to detect due to the scarcity of particles visible near those structures. Additional in vivo experiments and numerical simulations are needed to assess the effects of flow reversals on filtration in suspension-feeding fishes.

We thank M. Patterson and H. Burrell for aid in figure preparation; S. West, Z. Stroud and J. Viehweg for assistance in maintaining the fish; Perry Minnow Farm, Inc. for supplying the specimens; and P. Heideman, G. Capelli and C. Bagdassarian for comments on the manuscript. W.T.C. conducted this research in partial fulfilment of the requirements for the degree of BSc (Honors in Biology) from the College of William and Mary. The experiments were approved by the Research on Animal Subjects Committee, College of William and Mary, Project Number 0108. This research was supported by NSF grants IBN-9458114 and IBN-0131293 to S.L.S.

\section{References}

Ballintijn, C. M. (1969). Movement pattern and efficiency of the respiratory pump of the carp (Cyprinus carpio L.). J. Exp. Biol. 50, 593-613.

Beveridge, M. C. M., Sikdar, P. K., Frerichs, G. N. and Millar, S. (1991). The ingestion of bacteria in suspension by the common carp Cyprinus carpio L. J. Fish Biol. 39, 825-831.

Brainerd, E. L. (2001). Caught in the crossflow. Nature 412, 387-388.

Brewster, M. E., Chung, K.-Y. and Belfort, G. (1993). Dean vortices with wall flux in a curved channel membrane system. 1. A new approach to membrane module design. J. Membrane Sci. 81, 127-137.

Drucker, E. G. and Jensen, J. S. (1991). Functional analysis of a specialized prey processing behavior: winnowing by surfperches (Teleostei: Embiotocidae). J. Morph. 210, 267-287.

Finger, T. E. (1988). Sensorimotor mapping and oropharyngeal reflexes in goldfish, Carassius auratus. Brain Behav. Evol. 31, 17-24.

Finger, T. E. (1997). Feeding patterns and brain evolution in ostariophysan fishes. Acta Physiol. Scand. 161(Suppl. 638), 59-66.

García-Berthou, E. (2001). Size- and depth-dependent variation in habitat and diet of the common carp (Cyprinus carpio). Aquat. Sci. 63, 466-476.

Gerking, S. D. (1994). Feeding Ecology of Fish. San Diego: Academic Press. Goodrich, J. S., Sanderson, S. L., Batjakas, I. E. and Kaufman, L. S. (2000). Branchial arches of suspension-feeding Oreochromis esculentus: sieve or sticky filter? J. Fish Biol. 56, 858-875.

Hoogenboezem, W., Lammens, E. H. R. R., MacGillavry, P. J. and Sibbing, F. A. (1993). Prey retention and sieve adjustment in filter-feeding bream (Abramis brama) (Cyprinidae). Can. J. Fish. Aquat. Sci. 50, 465-471.

Hoogenboezem, W., van den Boogaart, J. G. M., Sibbing, F. A., Lammens, E. H. R. R., Terlouw, A. and Osse, J. W. M. (1991). A new model of particle retention and branchial sieve adjustment in filter-feeding bream (Abramis brama, Cyprinidae). Can. J. Fish. Aquat. Sci. 48, 7-18.

Lammens, E. H. R. R. and Hoogenboezem, W. (1991). Diets and feeding behaviour. In Cyprinid Fishes: Systematics, Biology and Exploitation (ed. I. J. Winfield and J. S. Nelson), pp. 353-376. London: Chapman and Hall.

Matthes, H. (1963). A comparative study of the feeding mechanisms of some African Cyprinidae (Pisces, Cypriniformes). Bijdragen tot de Dierkunde Amsterdam 33, 3-35.

Osse, J. W. M., Sibbing, F. A. and van den Boogaart, J. G. M. (1997). Intraoral food manipulation of carp and other cyprinids: adaptations and limitations. Acta Physiol. Scand. 161(Suppl. 638), 47-57.

Sanderson, S. L., Cech, J. J., Jr and Patterson, M. R. (1991). Fluid dynamics in suspension-feeding blackfish. Science 251, 1346-1348.

Sanderson, S. L., Cheer, A. Y., Goodrich, J. S., Graziano, J. D. and Callan, W. T. (2001). Crossflow filtration in suspension-feeding fishes. Nature 412, 439-441.

Sanderson, S. L., Mort, M. E. and Cech, J. J., Jr (1998). Particle retention by non-suspension-feeding cyprinid fishes. Can. J. Fish. Aquat. Sci. 55, 861-868.

Sanderson, S. L., Stebar, M. C., Ackermann, K. L., Jones, S. H., Batjakas, I. E. and Kaufman, L. (1996). Mucus entrapment of particles by a suspension-feeding tilapia (Pisces: Cichlidae). J. Exp. Biol. 199, 1743-1756.

Sanderson, S. L. and Wassersug, R. (1993). Convergent and alternative designs for vertebrate suspension feeding. In The Skull, vol. 3, Functional and Evolutionary Mechanisms (ed. J. Hanken and B. K. Hall), pp. 37-112. Chicago: The University of Chicago Press.

Sibbing, F. A. (1988). Specializations and limitations in the utilization of food resources by the carp, Cyprinus carpio: a study of oral food processing. Env. Biol. Fish. 22, 161-178.

Sibbing, F. A. (1991). Food capture and oral processing. In Cyprinid Fishes: Systematics, Biology and Exploitation (ed. I. J. Winfield and J. S. Nelson), pp. 377-412. London: Chapman and Hall.

Sibbing, F. A., Osse, J. W. M. and Terlouw, A. (1986). Food handling in the carp (Cyprinus carpio): its movement patterns, mechanisms and limitations. J. Zool. Lond. A 210, 161-203.

Sibbing, F. A. and Uribe, R. (1985). Regional specializations in the oro- 
pharyngeal wall and food processing in the carp (Cyprinus carpio L.). Neth. J. Zool. 35, 377-422.

Sobey, I. J. (1980). On flow through furrowed channels. Part 1. Calculated flow patterns. J. Fluid Mech. 96, 1-26.

Stairmand, J. W. and Bellhouse, B. J. (1985). Mass transfer in a pulsating flow with deposition onto furrowed walls. Int. J. Heat Mass Transfer 27, 1405-1408.
Summers, A. P. and Ferry-Graham, L. A. (2001). Ventilatory modes and mechanics of the hedgehog skate (Leucoraja erinacea): testing the continuous flow model. J. Exp. Biol. 204, 1577-1587.

Van den Berg, C., van Snik, G. J. M., van den Boogaart, J. G. M., Sibbing, F. A. and Osse, J. W. M. (1994). Comparative microanatomy of the branchial sieve in three sympatric cyprinid species, related to filter-feeding mechanisms. J. Morph. 219, 73-87. 